\title{
DEVELOPING CULTURE OF INQUIRY IN COMPUTER-SUPPORTED COLLABORATIVE LEARNING ${ }^{1}$
}

\author{
Lasse Lipponen \\ Department of Psychology \\ University of Helsinki \\ P.O. Box 13 \\ SF-00014 University of Helsinki \\ Finland \\ Email:lasse.lipponen@ helsinki.fi \\ tel: $+358-40-5563630$ \\ fax:+358-9-19123443
}

\author{
Kai Hakkarainen \\ Department of Psychology \\ University of Helsinki \\ P.O. Box 13 \\ SF-00014 University of Helsinki \\ Finland \\ Email:kai.hakkarainen@ helsinki.fi \\ tel: $+358-50-5634248$ \\ fax: $+358-9-19123443$
}

\begin{abstract}
The purpose of the study is to report results from an early stage of a longitudinal research on CSILE's (Computer-supported Intentional Learning Environments) cognitive effects in a Finnish elementary school. The study was aimed at analyzing how elementary school students' process of inquiry mediated by the CSILE environment developed from first (third grade) to second (fourth grade) year of CSILE use. The study was carried out by analyzing qualitatively 26 students' written productions in CSILE's database as well as by conducting some structured interviews. The study indicated that the students made a considerable progress in terms of explanation-seeking research questions, explanatory level of knowledge produced by them as well as quality of discourse interaction. However, at this point of time there were no indications of in-depth knowledge advancement that characterize the most successful Canadian CSILE classes. It is argued that facilitation of higher-level practices of inquiry in the context of the CSCL require a lot of pedagogical and epistemological support for both the teachers and students. It seems also to be important to engage a whole school community to the process of pedagogical development rather than deal with individual teachers and classrooms.
\end{abstract}

\section{INTRODUCTION}

The purpose to the study is to present results from an early stage of a four year longitudinal study focused on analyzing cognitive effects of computer-supported collaborative learning (the CSCL). The technological infrastructure of the study was provided by the Computer-supported Intentional Learning Environments (CSILE) developed by Scardamalia and Bereiter (1991). CSILE is a networked learning environment for fostering higher-level processes of inquiry in elementary-level education (see Scardamalia \& Bereiter, 1994). CSILE provides students a shared space for working productively with knowledge. The system is used to organize students learning projects in the context of such domains of knowledge as mathematics, physics, biology, and history. CSILE fosters participation in research-like process of inquiry at school by engaging students in a process of generating their own research questions, setting up intuitive theories and searching scientific information as well as sharing their cognitive achievements.

CSILE provides an advanced technological infrastructure as well as well-articulated cognitive practices for the CSCL. However, it seems that in order to effectively exploit new technology-based learning environments and corresponding cognitive practices, more systematic research on constraints and

\footnotetext{
1 The present study is a part of the Educational Technology Project of Helsinki (Helsinki 2001 project) coordinated by the Finnish IT Center for Schools, Vantaa Institution for Continuing Education, University of Helsinki. Finnish research on the CSILE environment is based on a research agreement between the Centre for Applied Cognitive Science, Ontario Institution for Studies in Education and Department of Psychology, University of Helsinki.
} 
conditions of successful implementation of the CSCL should be carried out. There is not available sufficient information concerning how CSILE fits in different school environments and how teachers with varying pedagogical experience and domain of expertise are able to function in CSILE-based learning environments. Experiences from the Canadian CSILE studies indicated that traditional pedagogical practices change very slowly, and a new culture of inquiry emerge only gradually through exploration and testing of new tools and practices (see, for example, Hakkarainen, 1997). A particularly important question is what kind of guidance do the students (and the teachers) need in order to productively use new collaborative tools provided by CSILE and corresponding learning environments.

These problems arose in the context of an ongoing four-year longitudinal study concerning CSILE's cognitive effects in Finnish elementary school. The purpose of the present study was analyze how elementary school students process of inquiry mediated by the CSILE environment evolved from first to second year of CSILE use. Results of the Finnish CSILE experiment are discussed by comparing those with results from Canadian CSILE studies. ${ }^{2}$ Further, out of the Finnish experiences seem to emerge certain constraints and condition of successful implementation of the CSCL.

\section{METHOD}

The study was carried out by setting up a CSILE network in a grade three (9 years old students) classroom in an elementary school from the City of Helsinki. The same group of 26 students is going to work with CSILE over the whole four years period. ${ }^{3}$ At the time of carrying out the study, the students had used CSILE about three terms, and were finishing the fourth grade. The school in question is an ordinary public elementary school without a long tradition of pedagogical development projects. The teacher assigned to the CSILE class is an expert of educational technology (providing technical support for the teachers in the whole district) but did not have extensive pedagogical experience. During the first year of the study, the students were allowed to use CSILE without any intervention by the researchers in

2 While comparing Canadian and Finnish CSILE students it should be taken into consideration that the Finnish students start school at age seven so that they had one year less experience of education that their Canadian peers. Further, while the Canadian CSILE studies usually focus on grade 5/6 students, the Finnish experiment was started from grade three. 3 The longitudinal study is based on the matchedpair design. However, the control material is not yet available. order to get information about the basic level of knowledge processing in Finnish elementary schools. During the second year, the funding of the research project allowed one researcher to visit weekly in the school, but not to provide extensive education or support for the teacher or the school.

During the first year studied, the Finnish CSILE students conducted several study projects focused on their familiar cultural and biological environment such as My Home Town Helsinki, Finland and North Haaga (i.e., their own suburb) as well as Dinosaurs and Solar System. During the second year, they carried out four study projects; 1) Northern Countries focused on studying Scandinavian countries, 2) Natural Phenomena focused on exploring some phenomena such as thunder or rain; 3) Ecology focused on environmental studies, and 4) Human focused on basic biological processes connected with human survival. The projects were initiated by the teacher and there was only a minimal amount of direct intervention from the researchers.

The study was carried out by analyzing qualitatively knowledge produced by the students to CSILE's database (see Hakkarainen, 1995; 1997 about the method). For the qualitative content analysis the notes were partitioned into ideas, i.e., a set of propositions that formed a coherent unit of meaning. 4 Reliability of the classification was assessed by using two independent raters and varied between .73 and .88 across variables used. The categories of knowledge analyzed were CSILE students' research questions, intuitive explanations, scientific information searched by them, and written communication, mediated by the CSILE environment, between the students. During the first year of CSILE use the students $(\mathrm{N}=26)$ produced 418 written comments, 422 research questions and 390 notes representing their own theories or new scientific information. During the second year they produced 619 comments, 150 research questions and 341 knowledge notes. The frequency data produced by the qualitative content analysis was analyzed by mean proportions of different categories of content in the context of an individual student. Evolution of CSILE students' culture of inquiry as well as effects of CSILE experience were analyzed by comparing an individual student's process of inquiry in his or first and second year of CSILE use. Furthermore, structured interviews were used to obtain detailed information about the students' conceptions of their own inquiry.

\footnotetext{
${ }^{4}$ In the following CSILE students' ideas classified will be referred as notes for practical reasons.. Due to the partitioning the number if ideas was about 1.5 times larger that notes because many notes consisted in more than one idea.
} 


\section{RESULT}

\subsection{Participation in CSILE use}

The result analysis indicated that computer-supported collaborative learning within the CSILE network was particularly motivating for Finnish male students who have traditionally been having a lot of difficulties with adapting to externally regulated traditional school learning. During the first year, the male students $(n=13)$ produced on average 78.3 CSILE notes $(S D=83.8)$ and female students $(n=$ 12) produced 17.4 notes $(S D=9.61) \quad(t=2.58$, $d f=12.40, \quad p<.024)$. The difference is partially explained by the fact that the CSILE teacher allowed the students to start working with CSILE after they had finished their other compulsory school assignments. As a consequence, 4 male students (two below average and two above average students) tried to finish their other assignments in the beginning of the week in order to get the rest of the week free for working with CSILE. Each of these male students produced over 100 notes to the database. It is remarkable, that the two below average students who represent avoidance orientation as their socioemotional coping strategy, were so actively participating in CSILE studies (see Järvelä, Hakkarainen, Lipponen, Niemivirta, \& Lehtinen, 1997). According to the estimation of the teacher, several below average male students were significantly improving their writing skills by using CSILE.

During the second year of CSILE study, the gender difference concerning participation in CSILE study was not as strong as in the first year; male students produced on average 50.7 notes $(S D=69.0)$ and female students 34 notes $(S D=49.7)$. This effect was not only caused by an increased female participation but also because the teacher controlled the male students participation and emphasized practice of basic skills of reading, writing, and math.

\subsection{Practices of Knowledge Processing}

Examination of knowledge produced by the Finnish CSILE students during their first year of CSILE use revealed that about $90 \%$ of research questions ( $f=$ 422) generated by the students were fact-seeking in nature (i.e., what, where, when and how many questions). This provided a strong contrast to the Canadian CSILE students who were systematically producing explanation-seeking why- and howquestions (Hakkarainen, 1995; 1997). A paired sample t-test was performed in order to test whether the mean proportion of explanation-seeking research questions increased from the first to second year of CSILE study. The analysis revealed that while the mean proportion of explanation-seeking research question was $0.11(S D=.22)$ during the first year of CSILE use it increased to $61(S D=.37)$ during the second year $(t=-6.59, \quad d f=25, \quad p<.000)$. Simultaneously, however, the number of questions generated decreased.

Further, approximately $50 \%$ of the knowledge notes $(f=390)$ produced during the first year were regarded by the researchers to be directly copied from books or some other sources of information. During the second year, a special consideration was given to guide the students to use scientific information in a more transformative way. The students were prevented from taking books and other information sources with them to the computers, and each student was provided with a special notebook for making his or her own notes while searching information ${ }^{i}$. An analysis of knowledge produced during the second year indicated that the intervention was very effective and direct copying of information practically disappeared. Moreover, from the first to second year of CSILE use happened a significant increase in the level of explanation in the students' intuitive knowledge $(t=-$ $3.06, d f=25, p<.005)$. While the mean level of explanation, as measured by a five-step scale from pieces of facts to integrated explanation, was 2.12 $(S D=.61)$ during the first year, it was $2.6(S D=.63)$ during the second year of the experiment.

Further, a structured interview was carried out in the beginning of the second year in order to assess each students' epistemological awareness, i.e., his or her conceptions of knowledge and inquiry. Examination of the results of the interview indicated that the changes in CSILE students' inquiry can be attributed to the intervention rather than a normal age-related development. The students were asked to assess different types of questions, knowledge, and comments produced by CSILE students from another Finnish CSILE school. Remarkably, only a part of the students regarded copying of knowledge as something to be avoided; many of them evaluated a directly copied piece of factual information to be more valuable than a student's own theory. They were also frequently preferring fact-seeking questions over explanation-seeking questions because the former are "easier to answer". After the interview a classroom discussion concerning cognitive value of different questions and practices of knowledge production was initiated. As a consequence, the students were increasingly construct explanation-seeking questions in their CSILE studies as well as other studies and started to prefer explanatory knowledge over factual information. Thus, it can be said that there happened a significant transition in epistemological nature of the students' inquiry from fact-centered to a more

\footnotetext{
i This method was suggested by Carl Bereiter.
} 
explanation-centered one during the first two years of the study. However, the students did not, at this point of the experiment, engage in a same kind of indepth knowledge advancement than has been reported in earlier CSILE studies (see, for example, Hakkarainen, 1997).

\subsection{Practices of Interaction}

Analysis of written communication mediated by the CSILE network revealed that during the first year of CSILE use the object of students' communication was factual information in $73.4 .6 \%(f=307)$ of all comments. While discourse interaction in the first year focused mostly on factual questions and answers, new form of discourse emerged during the second year. First, the mean proportion of explanationrelated comments increased from $.03(S D=0.06)$ to $.19(S D=.24)(t=-3.25, d f=24, p<.003)$. Second, the students started to discuss about methods and principles of their inquiry. While mean proportion of comments related to research methods was $.01(S D=$ $.03)$ during the first year, it was $.10(S D=.15)$ during the second year $(t=-2.95, d f=24, p<.007)$. Even if the proportions do not appear to be very high, these types of comments represent an emergence of a more developed discourse interaction that has a potential to facilitate advancement of the students' inquiry. During the second year emerged a rather heated discussion in which many students criticized comment that were exclusively focused on linguistic form. Comments on research methods represented metadiscourse that helped the learning community to regulate its practices of inquiry (see Hakkarainen, 1997).

Examination of CSILE students communicative ideas during the first year indicated that in many cases the students did not explicate referential relations of their comments. In written communication the referential relations should be explicated and the context created while those are usually known to participants in faceto-face communication. An analysis of CSILE students network-mediated communication revealed that while the mean level of explication, as assessed by a four-step scale, was $2.02(S D=1.07)$ during the first year, it was $2.73(S D=.63)$ during the second year $(t=-2.77, d f=22, p<.011)$. The change was apparently facilitated through comments in which the students requested each other to explicated referential or explanatory relations of their comments.

\section{DISCUSSION}

In the present study was reported results from the first two years of a four-year longitudinal study on CSILE cognitive effects. Experiences of the present study indicate that constraints and conditions of successful implementation of the CSCL should be carefully examined in order to implement cognitive ideals in educational practice. The study suggested that the content of curriculum in an early elementary level constrains possibilities of implementing higher-level practices of inquiry. Traditional curriculum starting from the students' immediate familiar environment is neither very motivating nor provides conceptually challenging problems for the students to explore. In the case of the present study, the authors attempts to design study projects focused on conceptually challenging problems of nature or history were rejected on the ground that the curriculum does not allow pursuing of these topics at this grade level. Further, experiences of the Finnish CSILE project (including several schools) indicated that elementary school teachers' subject domain expertise might considerably constrain more advanced forms of inquiry. As a consequence, the teachers are reluctant to start conceptually challenging projects in the science domain that would provide good opportunities for the students to engage in authentic processes of inquiry. Solution of the problem seems to require considerable changes in teacher education as well as engagement of external domain experts with elementary school students' study projects.

Cognitive research on educational practice aims at facilitating research-like process of inquiry in which generation of the students' own research questions, intuitive theories and search of explanatory scientific knowledge play an important role. A problem with implementing this kind of pedagogical practices at school is that teachers themselves have only seldom experience or epistemological knowledge concerning higher-level practices of inquiry. However, the students cannot be expected to discover these practices by themselves without guidance and expert modeling. It follows that in order to succeed the teachers might need a lot of epistemological support from researchers in the form of project designs, good examples, theoretical knowledge about inquiry and so on. The present authors are currently working with practically oriented guidelines that will provide support for the teachers and the students in engagement with higherlevel practices of inquiry.

CSILE's current version is based on a local-area network used within a classroom. It follows that there are usually only one or two CSILE classes in a school, and, therefore, the CSILE teachers are often working alone. However, it requires a lot of determination and pedagogical expertise from one teacher to carry out profound pedagogical changes without support from his or her colleagues. In order to overcome this constraint it seems necessary to engage a whole school community in work for pedagogical development, and carry out innovative study projects parallelly in networked and conventional classrooms. The next-generation CSILE technology, Knowledge Forum (Lea \& Scardamalia, 
1997), allows networking of a whole school instead of one or two classes, providing new tools for a school-wide pedagogical change. While preparing the third year of the Finnish CSILE experiment, the experimental school has formed, in collaboration with the present authors, a larger group of teachers who is committed to work for same kind of pedagogical changes in their classrooms as the CSILE class has been carrying out either by using the CSILE technology or conventional methods.

Although the Finnish CSILE project has not yet achieved epistemologically sophisticated and extraordinary processes of inquiry that characterize the Canadian CSILE studies (Scardamalia \& Bereiter, 1994; Lamon, Secules, Petrosino, Hackett, Bransford, \& Goldman, 1996), the project can be regarded as a relatively successful one: an inexperienced teacher in a school without extensive tradition of pedagogical innovations succeeded to engage students in highly motivating processes of inquiry and partially overcome certain basic constraints of Finnish educational culture such as fact-oriented educational epistemology. The most important lesson learned is that educational practices cannot be expected to change very quickly; it is a very complex and laborious process in which interaction between teachers, students and researchers plays a crucial role.

\section{REFERENCES}

Hakkarainen, K. (1995) Collaborative inquiry in the Computer-supported intentional learning environments. A poster presented in the Annual conference of the European Association for Research on Learning and Instruction. University of Nijmegen. August 26 to 31, 1995.

Hakkarainen (1997) Knowledge-seeking inquiry and computer-supported collaborative learning. A draft of Ph.D. thesis to be submitted to the University of Toronto.

Järvelä, S, Hakkarainen, K., Lipponen, L., Niemivirta, M., \& Lehtinen, E. (1996) The interaction of students' motivational orientation and cognitive processes in CSILE-based learning projects. A paper to be presented at the symposium under the title: Computer-support for collaborative learning: advancements and challenges. The 7th Conference for Research on Learning and instruction, August 26-30, 1997, Athens, Greece.

Lamon, M., Secules, T., Petrosino, A., Hackett, R., Bransford, J., \& Goldman, S. (1996) Schools for thought: Overview of the international project and lessons learned from one of the sites. Schauble, L. \& Glaser, R. (Eds.) Contributions of instructional innovation to understanding learning. Hillsdale, NJ: Erlbaum.

Lea, N. S. \& Scardamalia, M. (1997) Progressive curriculum \& knowledge building uses of networked multimedia resources. A paper presented at the Annual Meeting of the American Educational Research Association, Chicago, March 24, 1997.

Scardamalia, M., \& Bereiter, C. (1991) Higher levels of agency for children in knowledge building: A challenge for the design of new knowledge media. The Journal of The Learning Sciences, 1, 37-68.

Scardamalia, M., \& Bereiter, C. (1994) Computer support for knowledge-building communities. The Journal of the Learning Sciences, 3, 265283. 